\title{
Impacts of Chemical Abundances on Modeling Photoionization Case of Hydrogen and Helium
}

\author{
Belay Sitotaw Goshu \\ Department of Physics, College of Natural and Computational Science, Dire Dawa University, Dire Dawa, Ethiopia
}

Email address:

belaysitotaw@gmail.com

To cite this article:

Belay Sitotaw Goshu. Impacts of Chemical Abundances on Modeling Photoionization Case of Hydrogen and Helium. American Journal of Astronomy and Astrophysics. Vol. 5, No. 5, 2017, pp. 50-56. doi: 10.11648/j.ajaa.20170505.11

Received: September 25, 2017; Accepted: October 30, 2017; Published: January 19, 2018

\begin{abstract}
A number of models were utilized to study hydrogen and helium ionization structure by assessing the impacts of effective temperatures by different scholars. But this work mainly focused on two temperatures, $35000 \mathrm{~K}$ and $45000 \mathrm{~K}$ and enhancement of abundances. Each model is characterized based on the solar abundances and enhanced abundances by scale factors of 5. The photoionization code cloudy was used to construct the model of the nebulae. The result revealed that at high temperature $45000 \mathrm{~K}$, the sphere of helium close to hydrogen whereas at low temperature $35000 \mathrm{~K}$, hydrogen ion ratio is more dominant than helium and its radius is greater than it. Moreover, the chemical abundances ratio at each stage of ionization depends oneffective temperatures and enhancement of abundances.
\end{abstract}

Keywords: Photoionization, Chemical Abundances, Nebulae, Enhancement

\section{Introduction}

The ionization structure of elements can be determined using the chemical abundances. Hydrogen and helium are the most abundant elements and are called metals. The other elements like oxygen, sulfur, carbon, and neon are the next most important dominant elements. Since $\mathrm{H}$ and $\mathrm{He}$ are the most abundant elements, then the ionization structure of gaseous nebulae depends on it. The other heavy elements like, $\mathrm{O}, \mathrm{C}, \mathrm{Ne}$, and $\mathrm{N}$ have a significant contribution to the ionization structure, emission lines, and temperature fluctuations. Therefore the presence of these elements has a little significance on modeling of photoionization.

In order to solve this problem, we apply numerical models of gaseous nebulae using photoionization code of cloudy 90 [1]. Cloudy is one of the most powerful photoionization codes available today; it is extensively and clearly explained, and offers the charge to explore many different aspects of the physical process over a wide range of parameters. By applying cloudy, it has been possible to be more reliable numbers for the chemical composition and central star temperature of these objects, as well as better understanding the processes and the condition that limit the accuracy with which these quantities can be determined with the data that is available.

Chemical abundances of gaseous nebulae are among the most frequently used astrophysical tools for measuring the rates of heavy element formation, inferring the history of star formation and estimate the effects of interaction in the interstellar parts. Therefore, the purpose of this paper is to develop a model on the photoionization of gaseous nebulae to study the ionization structure of elements and the ratio of chemical abundances at each stage of ionization with the effective temperature and enhancement of abundances.

The structure of this paper is as follows. In section 2, we present the formulation of the problem with the basic assumption of the gaseous nebula. In section 3, we describe the model and the basic assumptions are discussed; this is followed by CLOUDY 90 [1]. In section 4, we present the results on the ionization structure elements and the last section we present the conclusions.

\section{Formulation of the Problem}

The starting point to tackle this problem is formulating ionization equilibrium equation. We assume that there is a constant temperature gas surrounding a spherically symmetric star provides a good approximation, which keeps the essentials of the situation without allowing unnecessary distractions. Each ion is assumed to be in ionization equilibrium, so the ionization rate for each ion is balanced by recombination. 
We also assume that the sole source of ionization is by absorption of radiation from the star giving a bound electron enough energy to escape from the atom. Recombination occurs when a free electron is captured by an ion with the creation of a photon. Since hydrogen is the most abundant element in the Universe, it will dominate in planetary formation, so we consider only a pure hydrogen interstellar medium at the beginning by taking a few elements of $\mathrm{O}, \mathrm{C}$, $\mathrm{N}, \mathrm{Ne}$ and $\mathrm{He}$ abundances relative to hydrogen atoms. Therefore, we restrict our self to the examination of photoionization equilibrium, which occurs only from the ground state of atoms.

The equation of transfer of radiation of intensity $I_{v}$ of a continuous spectrum and at a distance $r$ from the center of nebulae is written in the form

$$
\frac{d I_{v}}{d v}=-k_{v} I_{v}+j_{v}
$$

where $j_{v}$ is the volume emission coefficient, $k_{v}$ is the opacity is given by

$$
k_{v}=\sum N\left(x^{i}\right) a_{v}\left(x^{i}\right)
$$

where the summation is realized overall ions $x^{i}$ ( $\mathrm{x}$ is the element, $\mathrm{i}$ is the degree of ionization) and $a_{v}$ is the cross section of photo-ionization of $N\left(x^{i}\right)$. Assuming that the process of ionization and radiative recombination are in equilibrium, we have

$$
N\left(x^{i}\right) \int_{v_{o}}^{\infty} \frac{4 \pi J_{v}}{h v} a_{v} d v=N_{e} N\left(x^{i+1}\right) \alpha\left(x^{i}, T\right)
$$

where $a_{v}, o$ is the ionization threshold frequency, $\frac{4 \pi J_{v}}{h v}$ is the number of incident photons per unit area per unit time per unit frequency interval. $N\left(x^{i}\right), N_{e}, N\left(x^{i+1}\right)$ are the neutral atom, electron and proton densities per unit volume, and is the number of electron density and $\alpha\left(x^{i}, T\right)$ is the recombination coefficient; the right hand-side of the equation gives the number of recombination per unit volume per unit time.

The constraint equation of ions number density for each element $\mathrm{x}$ is

$$
N(x)=N\left(x^{o}\right)+N\left(x^{1}\right)+\cdots+N\left(x^{i}\right)
$$

We can solve for the ionization structure of gaseous nebula by coupling Equations (3) and (4). The ionization ratio ofelement is given by

$$
\frac{N\left(x^{i+1}\right)}{N\left(x^{i}\right)}=\frac{\int_{v_{o}}^{\infty} \frac{4 \pi J v}{h v} a_{v} d v}{N_{e} \alpha\left(x^{+i}, T\right)}
$$

where $J_{v}$ is the mean photon intensity of ionizing radiation at the point and is given by

$$
4 \pi J_{v}=\frac{4 \pi R^{2}}{4 \pi r^{2}} \times \pi F_{v}(0) e^{-\tau_{v}}=\frac{L_{v}}{4 \pi r^{2}}
$$

and $\alpha\left(x^{+i}, T\right) \mathrm{i}$; Te) is the radiative recombination coefficient and is given by

$$
\alpha\left(x^{+i}\right)=\sum_{n=1}^{\infty} \alpha_{n}
$$

where $\alpha\left(x^{+i}, T\right)$ is the recombination coefficient at the electron temperature Te, $\alpha\left(x^{+i}, T\right)$ in $\left(\mathrm{cm}^{3} \mathrm{~s}^{-1}\right), \mathrm{R}$ is the radius of thestar, $r$ is the distance from the source, $r$ is the optical depth, $L_{v}$ is the luminosity of the star per unit frequency interval, $\pi F_{v}$ is the flux density at the surface of the star and $\alpha_{n}$ are the coefficient recombination to level $\mathrm{n}$ and $v_{n}$ is the corresponding threshold frequency. The electron number density is considered to be given by

$$
N_{e}=N\left(H^{+}\right)+N\left(H e^{+}\right)
$$

where $\mathrm{Ne}, \mathrm{N}\left(\mathrm{H}^{+}\right)$, and $\mathrm{N}\left(\mathrm{He}^{+}\right)$are densities of electron, hydrogen and helium ions respectively. The flux density at the surface of the star is given by [2].

$$
\pi F_{v}=\frac{2 h v^{2}}{c^{2}} \frac{1}{e^{\frac{h v}{K T}}-1}
$$

where $\mathrm{h}$ is Planck's constant, $\mathrm{c}$ is the velocity of light, and $\mathrm{k}$ is the Boltzmann's constant.

Basic Assumption:

Before we present the photo-ionization model of the gaseous nebula, we make the basic assumptions and approximations for this process.

1. We take only radiation energetic for most of the atom enough to ionize hydrogen $(h v \geq 13: 6 \mathrm{eV})$.

2. All ions are in the ground state when they absorb radiation or recombine with an electron. This is one of the standard nebular approximations.

3. We ignore any dynamics, turbulence and that effect of shocks and ionizations fronts.

4. We focus on the abundances of the stages of ionization of $\mathrm{H}, \mathrm{He}, \mathrm{C}, \mathrm{N}$, and $\mathrm{O}$ that have ionization potential $\geq$ $\chi\left(H^{0}\right)$ and $\chi((\mathrm{He})=54.4 \mathrm{eV}$.

5. Photoionization equilibrium with the central star can have a black body distribution in frequency models having effective temperature $35000 \mathrm{~K}$ have the ionizing spectrum cut off above 4Ryd $(1 \mathrm{Ryd}=13.6 \mathrm{eV})$ and its radius of the star is around $\log (r)=10.75$.

6. The ionization structure of $\mathrm{H}$ and $\mathrm{He}$ is computed lonthe-spot" (OTS) approximation. This is because He II recombination to the ground level of $\mathrm{He} \mathrm{I}$ as the ionizing source of and $\mathrm{Ho}$ and $\mathrm{He}$ as well as the recombination to excited levels of $\mathrm{He}$ as ionizing sources of Ho

7. We assumed uniform hydrogen number density Log ( $\mathrm{n}$ $(\mathrm{H}))=4.2 \mathrm{~cm}^{-3}$ and the filling factor of the volume filled by nebulae is assumed to be uniform throughout the nebulae i.e., unity.

8. The element is considered for the nebulae are $\mathrm{He}, \mathrm{C}, \mathrm{N}$, $\mathrm{O}$, and Ne. These elements are considered as the primary elements.

\section{Photoionization Model of Gaseous Nebulae}

When we are close to the source, the density of ionizing photons is large. Therefore, the resulting ionization state of the gas is higher. If there are enough gaseous nebulae, all the 
ionizing photons can be absorbed and produce ionization bounded nebulae. If not, the nebulae are called density bounded. The density is constant throughout the region without changing its magnitude.

In an ionization bounded nebula purely composed of hydrogen, the total number of recombination per unit time balances the total a number of photons with energies. The total number of photons with energies above 13:6 eV emitted per unit time either by the star or during recombination to the ground level.

Table 1. The logarithm of numbers relative to hydrogen atom per $\mathrm{cm}^{-3}$.

\begin{tabular}{ll}
\hline Element & Abundances $(\log \mathbf{X i} / \mathbf{H})$ \\
\hline Helium & -1.00 \\
Carbon & -3.61 \\
Nitrogen & -4.07 \\
Oxygen & -3.31 \\
Neon & -4.00 \\
\hline
\end{tabular}

We focused on a description of how cloudy was used to obtain ionization structure of elements with the relative abundances of heavy metals given by [3, 4], which is shown in Table 1. In order to analyze the ionization structure of elements, we use the abundances elements shown in Table 1 and we compare these results with the abundances are enhanced by scale factors 5 .

Photoionization codes can solve the equations of ionization and thermal equilibrium for both gas and dust particles. This allows the determination of the ionic fractions, the electron temperature and the line and continuum emissivity in each cell of the model. In this works, we mainly focus only on the equilibrium of gas, not dust. This can be easily calculated from photoionization codes to understand knowledge of gas photon interactions in the interstellar medium [5] to explain the ionization structure of hydrogen and helium in two different cases.

The region is assumed to be a static sphere, completely surrounding the central star and letting no inwarddirecteddiffuse line or continuum radiation escape. The inner radius, $\mathrm{Ro}$, is left as a free variable, with $\log (\mathrm{Ro})=16.7$ to cover the range of observed radii gaseous nebulae. The value of the inner radius and the extension of the ionized region are basically determined by the total hydrogen density.

The Ionization parameter: We can define ionization parameter $\mathrm{U}$, as the number of ionizing photons per hydrogen atom. This quantity is determined by the central source and the distance $r$ to the central source. At the inner boundary of radius $R_{0}$, the ionization parameter is given by

$$
U=\frac{Q_{H}}{4 \pi R_{S}^{2} N(H) c}
$$

Where $\mathrm{Q}(\mathrm{H})$ is the number of ionizing photons emitted per hydrogen atom. $\mathrm{N}(\mathrm{H})$ is the number of hydrogen atom percm $^{-3}$ and $\mathrm{c}$ is the speed of light.

This expression is often used to specify the flux of incident continuum at the inner boundary of a cloud with a plane parallel geometry and is an important parameter determining the ionization structure of the cloud.
Cloudy stops when the temperature is less than 4000K [6], but in this work, we prefer to use the lowest temperature as $100 \mathrm{~K}$. This is due to our choice of references. Close examination in each model suggests that there is a distinct region which depends on the temperature [7]. This belongs to the class of cool because of enhancement of chemical abundances.

\section{Ionization Stages of Hydrogen and Helium}

We shall be concerned only with the pair of nebulae in which, hydrogen is ionized. Radiation with $v>v_{o}$, where $v_{o}$ is the hydrogen ground state ionization limit, will be referred to as ionizing radiation. The ionization potential of hydrogen is $I(H)$ $=13.6 \mathrm{eV}$. The photoionization of cross-section of hydrogen element depends on the ionization energy and given by

$$
a_{v}=a_{o} f\left(\frac{v}{v_{o}}\right)
$$

where $f\left(\frac{v}{v_{o}}\right)=\left(\frac{v}{v_{o}}\right)^{-3}$ and $a_{o}=6.3 \times 10^{-18} \mathrm{~cm}^{2}$. Where $v_{o}$ is the threshold frequency of hydrogen

Helium: The photoionization cross section of helium atom is given by

$$
a_{v}(H e)=a_{v o} e^{-0.73\left(v-1.807 v_{o}\right)}
$$

where $x=\frac{v}{v_{1}}, v=1.807 v_{o}=5.91 \times 10^{15} \mathrm{~Hz}$ is the threshold frequency of helium atoms at which it is ionized. The ionization structure of helium atoms per its neutral is described by

$$
\frac{N\left(H e^{+}\right)}{N(H e)}=\frac{\int_{1.807 v o}^{\infty} 4 \pi J_{v a_{v}}\left(H e^{+}\right) d v}{N_{e} \alpha_{B}\left(H e^{o}, T\right)}
$$

where $\alpha_{B}\left(H e^{o}, T\right)$ is the recombination coefficient of helium excited ions.

Heavy Elements: The ionization structure of heavy elements can be found with the ionization equilibrium equation for any two successive stages of ionization which described by [2] again the total number of ions of all stages of ionization is described by equation [12]. The total number of density is given by

$$
N\left(x^{i}\right)=\frac{N(x)}{1+R_{1}+R_{2} R_{3}+R_{1} R_{2} R_{3} \cdots}
$$

Where $R_{1}=\frac{N\left(x^{i}\right)}{N(x)}, R_{2}=\frac{N\left(x^{+2}\right)}{N\left(x^{+i}\right)}$ and $R_{3}=\frac{N\left(x^{+3}\right)}{N\left(x^{+2)}\right.}$ and then we can write the ratio of abundances of heavy elements given by

$$
\frac{N\left(x^{+m}\right)}{N(x)}=\frac{R_{1} R_{2} R_{3} \ldots \ldots}{1+R_{1}+R_{1} R_{2}+R_{1} R_{2} R_{3}+\cdots}
$$

The atomic parameters of heavy elements are given by [1, 8]. Ionization cross section of heavy species be complex due to its structure. We have a good interpolation formula that fits the contribution of each threshold $v_{T}$ of the photoionization cross section by 


$$
a_{v}=a_{T}\left(\beta\left(\frac{v}{v_{T}}\right)^{-s}+(1-\beta)\left(\frac{v}{v_{T}}\right)^{-1-s}\right)
$$

Equation (15) is true only for the frequencies greater than the threshold and the total cross section is then the sum of the contribution of the individuals threshold, where $\beta$ and $\mathrm{s}$ are already determined for each heavy elements and it is given by $[1,8,9,10,7$ and 11]. The ionization structure of heavy elements obtained from the ionization equilibrium and recombination coefficient

$$
\frac{N\left(x^{+}\right)}{N(x)}=\frac{\int_{v_{T}}^{\infty} 4 \pi J_{v a_{v}}(x) d v}{N_{e} \alpha_{G}\left(x^{o}, T\right)}
$$

where $\alpha_{G}\left(x^{o}, T\right)$ is the recombination coefficient of heavy trace elements. In this work, we assumed the recombination coefficient of helium atoms is constant throughout the radius. The ionization fraction of helium ions to helium atoms in the range of frequency of $5.93 \times 10^{15} \mathrm{~Hz}$ to $1.16 \times 10^{16} \mathrm{~Hz}$ or 24.6 $\mathrm{eV}$ to $54.4 \mathrm{eV}$

\section{Results and Discussions}

Photoionization models obtained with numerical codes are widely used to study the physics of the interstellar medium (Planetary Nebulae, HII regions, etc.). We vary the effective temperature and chemical abidance by changing with scale factors 5 of the most dominant elements. The principal parameters of the nebular gas are the hydrogen density and its distribution is uniform at $\log \mathrm{n}(\mathrm{H})=4.2 \mathrm{~cm}^{-3}$, and the filling factor is unity. In addition, the effective temperatures are 35000 and $45000 \mathrm{~K}$ and a set of chemical abundances shown in Table 1. The model is computed ionization structure abundances ratio at each stage of ionization is shown in Figures 1 and 2 respectively. The other results are shown in Tables 2, and 3.

Helium abundances determinations often require a correction for unseen neutral hydrogen or helium. When the star ionizing photons might not be sufficient to make the spheres for hydrogen and helium coincide. Two different situations are shown in Figures 1 and 2 shown below. Both models have identical physical properties except for the temperature of the ionizing radiation field and chemical abundances. The model shown in Figure 1, the ionization structure of both hydrogen and helium which are ionized by a spectrum of temperatures 35000 and $45000 \mathrm{~K}$. and chemical abundances are given by [1, $8,9,10,7$ and 11].

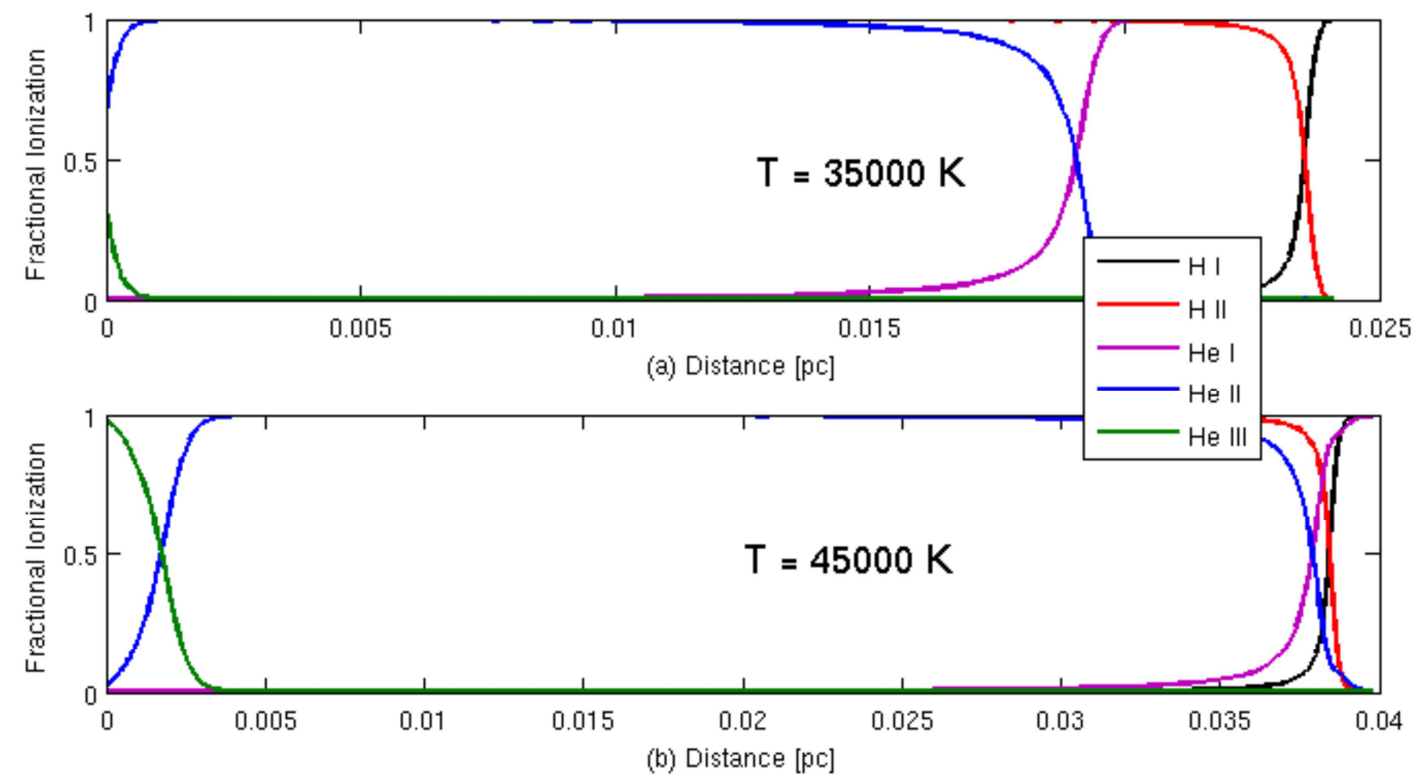

Figure 1. The Ionization Structure of hydrogen and helium at 35000 and $45000 \mathrm{~K}$ at the abundances of elements shown in Table1.

The upper panel is shown in Figure 1, the ionization fractions $\frac{H^{+}}{H}+$ and with $\frac{H e^{+}}{H}$ the nebulae ionized by a soft spectrum at temperature $35,000 \mathrm{~K}$, whereas the lower panel is ionized by $45000 \mathrm{~K}$. In these two models, the hydrogen abundance could overestimate since the radius of helium is smaller than that of hydrogen; we observed the same physical properties as in the lower panel. But the contribution of $\mathrm{He}^{2+}$ at the temperature of $35000 \mathrm{~K}$ is almost negligible relative to both $\mathrm{H}^{+}$and $\mathrm{He}^{+}$. This means the ionized energy is not enough to produce more ions of $\mathrm{He}^{+2}$ at this temperature. The reason is that there is no sufficient energy which is capable producing $\mathrm{He}^{+2}$ at this temperature. Instead, there is sufficient energy to ionize the second stage ionization of helium at this temperature. The reason is that, at higher temperatures, there are enough photons above $24.6 \mathrm{eV}$ to fully ionize helium, so that $\mathrm{x}(\mathrm{He}+) / \mathrm{x}(\mathrm{H}+)$ is dominant.

The ionization structure of hydrogen and helium of nebulae ionized with the same temperature as the previous results shown in Figure 1. The results are shown in the upper panel of Figure 2; the Stromgren radius of hydrogen is less than the previous results shown in Figure 1. As the abundances of chemical elements enhanced, the radius of 
hydrogen and helium is reduced. The same phenomena are observed at the second effective temperature $45000 \mathrm{k}$. The supply of helium is to make the sphere of both hydrogen and helium coincides at a distance. The second panel shows the radius of hydrogen and helium could not intersect this is due to the nature of the ionization structure of the other elements starts to dominate in the nebulae. We have produced hydrogen deficient nebulae in the interstellar medium.

We have checked the ratio of chemical abundances in each case. The results are shown in Tables 2 and 3 explain abundances ratio at each stage of ions and electron temperatures by changing the ionizing spectrum and enhancement of chemical abundances. Both these tables show how the effective temperature and enhancement effects, the chemical abundances of each ionization stages. Several interesting conclusions can be drawn from inspection of the Tables 2 and 3.
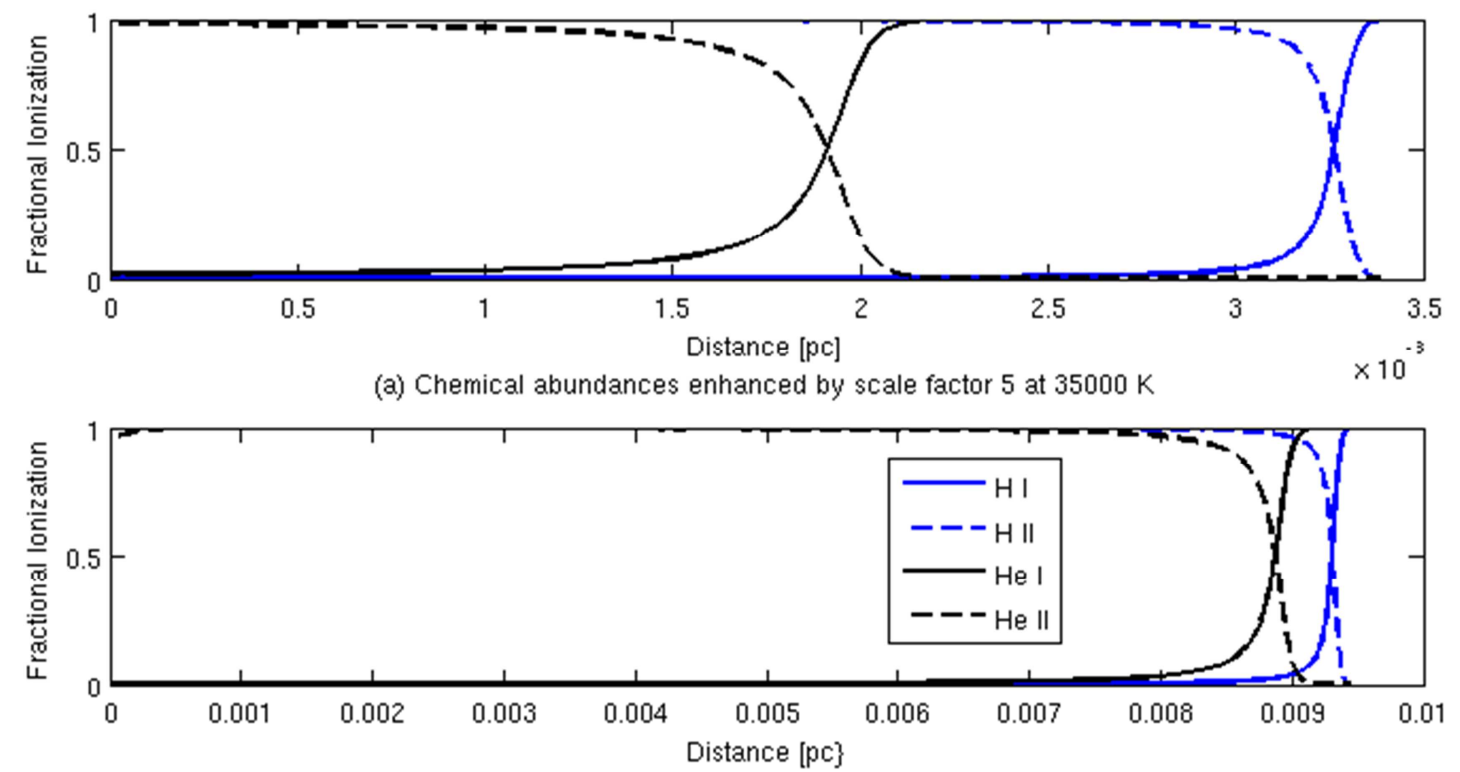

(b) Chemical abundances enhanced by scale 5 at $45000 \mathrm{~K}$

Figure 2. The Ionization Structure of hydrogen and helium at 35000 and $45000 \mathrm{~K}$ at the abundances of elements enhanced by scale factor 5.

Table 2. Ratio of Chemical abundances $X_{i} / H$ at each stages of ionization, electron temperature at the effective temperature of $35000 \mathrm{~K}$.

\begin{tabular}{|c|c|c|c|c|c|c|}
\hline \multirow[t]{2}{*}{ Element } & \multicolumn{4}{|c|}{ Ratio of Chemical Abundances } & \multirow[t]{2}{*}{$\mathrm{Ne}$} & \multirow[t]{2}{*}{ Te [K] } \\
\hline & 1 & 2 & 3 & 4 & & \\
\hline $\mathrm{H}$ & $1.79(-3)$ & $9.98(-1)$ & & & & \\
\hline $\mathrm{He}$ & $1.47(-2)$ & $9.85(-1)$ & $6.36(-4)$ & & $1.74(4)$ & $9.88(3)$ \\
\hline $\mathrm{C}$ & $7.04(-4)$ & $2.27(-1)$ & $7.72(-1)$ & $2.83(-4)$ & & \\
\hline $\mathrm{N}$ & $3.95(-4)$ & $3.23(-1)$ & $6.72(-1)$ & $3.57(-4)$ & & \\
\hline $\mathrm{O}$ & $1.23(-3)$ & $6.58(-1)$ & $3.40(-1)$ & $6.83(-5)$ & & \\
\hline $\mathrm{Ne}$ & $2.40(-3)$ & $8.20(-1)$ & $1.77(-1)$ & $2.26(-6)$ & & \\
\hline $\mathrm{H}$ & $2.30(-4)$ & $9.88(-1)$ & & & & \\
\hline $\mathrm{He}$ & $1.48(-2)$ & $9.81(-1)$ & $4.90(-4)$ & & $1.75(4)$ & $6.93(3)$ \\
\hline $\mathrm{C}$ & $1.43(-4)$ & $2.72(-1)$ & $8.28(-1)$ & $1.94(-4)$ & & \\
\hline $\mathrm{N}$ & $5.91(-4)$ & $3.73(-1)$ & $6.26(-1)$ & $2.82(-4)$ & & \\
\hline $\mathrm{O}$ & $1.65(-3)$ & $7.02(-1)$ & $2.98(-1)$ & $5.22(-5)$ & & \\
\hline $\mathrm{Ne}$ & $3.47(-3)$ & $8.56(-1)$ & $1.40(-1)$ & $1.36(-6)$ & & \\
\hline
\end{tabular}

We have checked the ratio of chemical abundances in each case. The results are shown in Tables 2 and 3 explain how the abundances ratio the elements at each stage of ionization and electron temperatures by changing the ionizing spectrum and enhancement of chemical abundances.

The upper part of Table 2 shows the abundances ratio of elements at different stages of ionization at normal abundances of elements, whereas the lower part shows when the abundances of the main elements are enhanced by scale factor 5. The result shows at the second stage of helium ionization, the ratio of helium abundance when it is enhanced by scale factor 5 , the ration is lowered by $0.4 \%$.
Similarly, the abundance ratio of hydrogen at the first and second stage of ionization is reduced by $87 \%$ and $20 \%$ and then we have hydrogen deficient nebulae because of the enhancement of the main elements. The abundances ratio of other elements at the second stages ionization is raised as it is shown in Table 2. The effective temperature of the electron is decreased by $29.9 \%$, whereas the electron density is raised by $0.5 \%$. This is due to enhancement processes of elements. This shows that the change of electron temperature is highly sensitive to the effective temperature of the ionizing spectrum whereas the electron density is not that much as electron temperature. 
Table 3. Ratio of Chemical abundances Xi/H at each stages of ionization, electron temperature at the effective temperature of $45000 \mathrm{~K}$.

\begin{tabular}{|c|c|c|c|c|c|c|}
\hline \multirow[t]{2}{*}{ Element } & \multicolumn{4}{|c|}{ Ratio of Chemical Abundances } & \multirow[t]{2}{*}{$\mathrm{Ne}\left(\mathrm{cm}^{-3}\right)$} & \multirow[t]{2}{*}{ Te [K] } \\
\hline & 1 & 2 & 3 & 4 & & \\
\hline $\mathrm{H}$ & $5.31(-4)$ & $9.99(-1)$ & & & & \\
\hline $\mathrm{He}$ & $1.63(-3)$ & $9.57(-1)$ & $4.16(-2)$ & & $1.75(4)$ & $1.03(4)$ \\
\hline $\mathrm{C}$ & $5.23(-6)$ & $3.53(-2)$ & $9.48(-1)$ & $1.71(-2)$ & & \\
\hline $\mathrm{N}$ & $9.15(-6)$ & $2.91(-2)$ & $9.46(-1)$ & $2.46(-2)$ & & \\
\hline $\mathrm{O}$ & $2.58(-5)$ & $2.04(-2)$ & $9.11(-1)$ & $1.85(-2)$ & & \\
\hline $\mathrm{Ne}$ & $5.10(-5)$ & $1.20(-1)$ & $8.78(-1)$ & $2.03(-2)$ & & \\
\hline $\mathrm{H}$ & $2.04(-1)$ & $9.59(-1)$ & & & & \\
\hline $\mathrm{He}$ & $2.08(-3)$ & $9.66(-1)$ & $3.20(-2)$ & & $1.76(4)$ & $6.81(3)$ \\
\hline $\mathrm{C}$ & $7.99(-6)$ & $4.62(-2)$ & $9.42(-1)$ & $1.17(-2)$ & & \\
\hline $\mathrm{N}$ & $1.55(-5)$ & $3.73(-2)$ & $9.43(-1)$ & $2.01(-2)$ & & \\
\hline $\mathrm{O}$ & $4.29(-5)$ & $9.34(-2)$ & $8.90(-1)$ & $1.63(-2)$ & & \\
\hline $\mathrm{Ne}$ & $1.029(-5)$ & $1.66(-2)$ & $8.32(-1)$ & $1.32(-3)$ & & \\
\hline
\end{tabular}

As it was shown in Table 2, Table 3 has two parts. The upper part of this table shows the ratio of chemical abundance at each stage of ionization before the main elements abundances are enhanced at the effective temperature of $45000 \mathrm{~K}$. Relative to the previous Table 2, the abundances of helium at the third stage of ionization, the ratio of $\mathrm{He}^{++} / \mathrm{H}^{+}$is larger than the result shown in Table 2. This is due to rise of the effective temperature in the nebulae, whereas at the second stage of ionization, the ratio of $\mathrm{He}^{+/} / \mathrm{H}^{+}$ is less than the results shown in Table 2 . The other results show that the ratio of each element at the third and fourth dominates relative of the previous results shown in Table 2. Similar to the result is shown in Table 2, the abundance hydrogen of hydrogen decrease.

At the lower panel of Table 3 shows when the abundances of main elements are enhanced by scale factor 5 . The results show that the ratio of helium abundance at the second stage of ionization is raised by $10 \%$ and the ratio at the third stage is decreased by $10 \%$. The other elements ionization ratio at the third and fourth stage is decreased and shifted to the second stage of ionization relative to the upper result shown in Table 3.

The electron temperature is also affected by the enhancement processes. When the chemical abundances of $\mathrm{C}, \mathrm{N}, \mathrm{O}$, and $\mathrm{Ne}$ are enhanced by scale factor 5 , the result shows that the electron temperature is decreased by $56.6 \%$ at the effective ionizing spectrum $35000 \mathrm{~K}$. In the second case, when the ionizing spectrum is increased to $45000 \mathrm{~K}$, the electron temperature decreases by $75.6 \%$. This shows that the change of electron temperature is highly sensitive to the effective temperature of ionizing spectrum. This is due to forbidden lines of oxygen and nitrogen which an impacts of lowering the electron temperature

\section{Conclusions}

The photoionization code cloudy was used to construct the model of the nebulae [1]. The basic assumption discussed in section III and the chemical abundances given by $[1,8,9,10$, 7 and 11]. and we develop the new model developed by enhancing the chemical abundances by changing the scale factors of main elements. In order to tackle this the problem we consider two selected temperature of $35000 \mathrm{~K}$ and 45 $000 \mathrm{~K}$ to study the ionization fraction of hydrogen and helium ions versus the optical depth. At low temperature, we find that hydrogen is much more ionized than helium at least in this parameter range so far which is explored by us. As it was shown by [12] the result of ionization from the excited states of $\mathrm{H}$ i. We have found the ratio of $\mathrm{Xi} / \mathrm{H}$ at each stage of ionization. The ratio of abundance at each stages of ionization depends on effective temperature and enhancement of chemical abundances.

At high temperature, $45000 \mathrm{~K}$, the supply of helium made the sphere of both hydrogen and helium closer to each other. Moreover, we also assessed by enhancing the abundances of chemical elements, the result shows that there is deficient of hydrogen concentration in the nebulae and as the result temperature of electrons falls. As it was shown by [13], the abundances of metal contents increase when the electron temperature decreases.

\section{References}

[1] Ferland G. J., et al., 2013, Hazy, Introduction to cloudy c13.04.

[2] Osterbrock, D. E. 1989, Astrophysics of gaseous nebulae and active galactic nuclei, University Science Books, 1989.

[3] Grevesse N and Sauval A. J. Standard Solar Composition. Space Science Reviews, 85:161-174, May 1998. doi: 10.1023/A:1005161325181.

[4] Asplund M., Grevesse N., Sauval A. J. and Scott P., 2009, ARRA, 47, 481.

[5] Morisset C., 2017, Planetary Nebulae: Multi-wavelength probes of stellar and galactic evolution, International Astronomical Union Proceedings IAU Symposium No. 323, 2017.

[6] Bohigas J., 2008, The Astrophysical Journal, 674, 95-975.

[7] Pilyugin L. S., Ve'lchez J. M., and Thuan T. X, 2010, The Astro- physical Journal, 720: 1738-1751.

[8] Tielens A. G. G. M., The physics and the chemistry of the interstellar medium, 2005, Cambridge university press, pp 240. 
[9] Holweger H., Photospheric Abundances: Problems, Updates, Implications. In R. F. Wimmer-Schweingruber, editor, Joint SOHO/ACE workshoplSolar and Galactic Composition", volume 598 of American Institute of Physics Conference Series, page 23, 2001. doi: 10.1063/1.1433974.

[10] Allende Prieto C., Lambert D. L., and Asplund M., the Forbidden Abundance of Oxygen in the Sun. ApJ, 556: L63L66, July 2001. doi: 10.1086/322874.
[11] Allende Prieto C., Lambert D. L. and Asplund M., A Reappraisal of the Solar Photospheric C/O Ratio. ApJ, 573: L137-L140, July 2002. doi: 10.1086/342095.

[12] Zsarg'o J., D. J. Hillier D. J., and Georgiev L. N., RevMexAA (Seriede Conferencias), 33, 58-58 (2008).

[13] Kinkel U and Rosa M. R., 1994, Astron. Astrophys, 282, L37L40. 